\title{
TOTAL CURVATURE OF COMPLETE SURFACES IN HYPERBOLIC SPACE
}

\author{
GIL SOLANES
}

\begin{abstract}
We prove a Gauss-Bonnet formula for the extrinsic curvature of complete surfaces in hyperbolic space under some assumptions on the asymptotic behaviour. The result is given in terms of the measure of geodesics intersecting the surface non-trivially, and of a conformal invariant of the curve at infinity.
\end{abstract}

\section{INTRODUCTION AND MAIN RESULTS}

In this paper we prove a Gauss-Bonnet formula for the total extrinsic curvature of complete surfaces in hyperbolic space. Our result is analogous to those obtained by Dillen and Kühnel in 2 for submanifolds of euclidean space, where the total curvature of a submanifold $S$ is given in terms of the Euler characteristic $\chi(S)$, and the geometry of $S$ at infinity (see also Dutertre's work [3] on semi-algebraic sets).

Our starting point is the following well-know equality for $S \rightarrow \mathbb{H}^{3}$, a compact surface with boundary immersed in hyperbolic 3-space:

$$
\int_{S} K d S=2 \pi \chi(S)+F(S)-\int_{\partial S} k_{g} d s
$$

being $K$ the extrinsic curvature of $S$ (i.e. the product of its principal curvatures), $F(S)$ the area, and $k_{g}$ the geodesic curvature of $\partial S$ in $S$. This formula follows from the classical (intrinsic) Gauss-Bonnet theorem, and the Gauss equation. We plan to make $S$ expand over a complete non-compact surface, but the last two terms in (11) are likely to become infinite. To avoid an indeterminate form, we add and subtract the area enclosed by the curve $\partial S$. Such a notion was defined by Banchoff and Pohl (cf. [1] and also [13]) for any closed space curve $C$ as

$$
\mathcal{A}(C):=\frac{1}{\pi} \int_{\mathcal{L}} \lambda^{2}(\ell, C) d \ell
$$

1991 Mathematics Subject Classification. 53C65.

Key words and phrases. Integral geometry, total curvature, hyperbolic space, open surfaces.

Work partially supported by FEDER/MEC grant number MTM2006-04353 and the Ramón y Cajal program. 
where $\mathcal{L}$ is (in our case) the space of geodesics in $\mathbb{H}^{3}$, $d \ell$ is the invariant measure on $\mathcal{L}$ (unique up to normalization), and $\lambda(\ell, C)$ is the linking number of $C$ with $\ell \in \mathcal{L}$. This definition was motivated by the Crofton formula which states

$$
F(S)=\frac{1}{\pi} \int_{\mathcal{L}} \#(\ell \cap S) d \ell
$$

where \# stands for the cardinal. Hence, we can rewrite (1) as follows

$$
\int_{S} K d S=2 \pi \chi(S)+\frac{1}{\pi} \int_{\mathcal{L}}\left(\#(\ell \cap S)-\lambda^{2}(\ell, \partial S)\right) d \ell+\mathcal{A}(\partial S)-\int_{\partial S} k_{g} d s .
$$

Our main result is a similar formula for complete surfaces in $\mathbb{H}^{3}$ defining a smooth curve $C$ in $\partial_{\infty} \mathbb{H}^{3}$, the ideal boundary of hyperbolic space. In that case, the last two terms of the previous equation are replaced by a conformal (or Möbius) invariant of the geometry of $C$ in $\partial_{\infty} \mathbb{H}^{3}$. To be precise, our result applies to surfaces with cone-like ends in the sense defined next. A similar notion of cone-like ends for submanifolds in euclidean space appears in [2].

Definition 1.1. Let $f: S \leftrightarrow \mathbb{H}^{3}$ be an immersion of a $\mathcal{C}^{2}$-differentiable surface $S$ in hyperbolic space. We say $S$ has cone-like ends if

i) $S$ is the interior of a compact surface with boundary $\bar{S}$, and taking the Poincaré half-space model of hyperbolic space, $f$ extends to a $\mathcal{C}^{2}$-differentiable immersion $f: \bar{S} \leftrightarrow \mathbb{R}^{3}$,

ii) $C=f(\partial \bar{S})$ is a collection of simple closed curves contained in $\partial_{\infty} \mathbb{H}^{3}$, the boundary of the model, and

iii) $f(\bar{S})$ is orthogonal to $\partial_{\infty} \mathbb{H}^{3}$ along $C$.

In particular, such a surface is complete with the induced metric. We will see that surfaces with cone-like ends have finite total extrinsic curvature. There are also examples of complete non-compact surfaces with finite total extrinsic curvature which do not fulfill $i$ ) or $i i$ ) in the previous definition. Condition $i i i$ ) however is necessary for the total curvature to be finite: the limit of the extrinsic curvature of $S$ at an ideal point $x \in C$ is $\cos ^{2}(\beta)$ where $\beta$ is the angle between $S$ and $\partial_{\infty} \mathbb{H}^{3}$ at $x$.

In the Klein (or projective) model, the definition reads the same, but replacing the word 'orthogonal' by 'transverse'. We will mainly work with the Poincaré half-space model. Unless otherwise stated all the metric notions (such as length, area or curvature) will refer to the hyperbolic metric.

Given a connected oriented curve $C \subset \partial_{\infty} \mathbb{H}^{3} \equiv \mathbb{R}^{2}$, and a pair of distinct points $x, y \in C$, let us consider the oriented angle at $x$ from $C$ to the oriented circle through $x$ that is positively tangent to $C$ at $y$. This angle admits a unique continuous determination $\theta: C \times C \rightarrow \mathbb{R}$ that vanishes on the diagonal. Note that $\theta(y, x)=\theta(x, y)$ and $\theta$ is independent of the orientation of $C$.

We will prove the following result. 
Theorem 1. Let $S \subset \mathbb{H}^{3}$ be a simply connected surface of class $\mathcal{C}^{2}$, embedded in the Poincaré half-space model of hyperbolic space, and with a (connected) cone-like end $C \subset \partial_{\infty} \mathbb{H}^{3}$. Then, the integral over $S$ of the extrinsic curvature $K$ is

$$
\int_{S} K d S=\frac{1}{\pi} \int_{\mathcal{L}}\left(\#(\ell \cap S)-\lambda^{2}(\ell, C)\right) d \ell-\frac{1}{\pi} \int_{C \times C} \theta \sin \theta \frac{d x d y}{\|y-x\|^{2}}
$$

where

- $d \ell$ is an invariant measure on the space of geodesics $\mathcal{L}$,

- $\lambda^{2}(\ell, C)$ is 1 if the ideal endpoints of $\ell$ are on different components of $\partial_{\infty} \mathbb{H}^{3} \backslash C$ and 0 otherwise, and

- $d x, d y$ denote length elements on $C$ with respect to the euclidean metric $\|\cdot\|$ on $\partial_{\infty} \mathbb{H}^{3} \equiv \mathbb{R}^{2}$.

The integrals in (3) are absolutely convergent.

Remark 1. The most interesting term in (3) is the last one, which we call the ideal defect of $S$. It defines a functional for plane curves which is invariant under the action of the Möbius group. In fact, the form $d x d y /\|y-x\|^{2}$, as well as $\theta(x, y)$, is invariant under Möbius transformations. Similar expressions for space curves appear often in the study of conformally invariant knot energies (cf. [5]).

The first term in the right hand side of (3) is positive, and can be considered as a 'truncated area' of $S$, in view of (2). We call this term the measure of non-trivial geodesics of $S$. From Proposition [6, it will be clear that it is a natural functional of $S$.

The idea of the proof is roughly the following. We pull-back $d \ell$ to the space of point pairs of $S$. Integration gives the measure of non-trivial geodesics. Applying Stokes' theorem yields then the result. This procedure was already used by Pohl in the euclidean setting in [8], but here we use a different 'primitive' of $d \ell$. This leads to a somehow dual construction, where the total curvature instead of the area appears. This dual approach is not possible in euclidean space.

From Theorem 1 one gets easily a formula for a general surface with cone-like ends.

Corollary 2. Let $S \rightarrow \mathbb{H}^{3}$ be a $\mathcal{C}^{2}$-immersed complete surface with cone-like ends $C_{1}, \ldots, C_{n}$, the curves $C_{i}$ being simple and closed. Then

$$
\begin{aligned}
\int_{S} K d S=2 \pi(\chi(S)-n)+\frac{1}{\pi} \int_{\mathcal{L}}\left(\#(\ell \cap S)-\sum_{i=1}^{n} \lambda^{2}\left(\ell, C_{i}\right)\right) d \ell & \\
& \quad-\frac{1}{\pi} \sum_{i=1}^{n} \int_{C_{i} \times C_{i}} \theta \sin \theta \frac{d x d y}{\|y-x\|^{2}}
\end{aligned}
$$

and the previous integrals are absolutely convergent. 
Proof. Take a compact set $K \subset \mathbb{H}^{3}$ with $\mathcal{C}^{2}$ boundary $\partial K$ transverse to $S$, and such that $S \backslash K=S_{1} \cup \ldots \cup S_{n}$, where each $S_{i}$ is an embedded topological cylinder over $C_{i}$. Applying (11) and (2) to $R=S \cap K$ yields

$$
\int_{R} K d R=2 \pi \chi(R)-\int_{\partial R} k_{g}(s) d s+\frac{1}{\pi} \int_{\mathcal{L}} \#(\ell \cap R) d \ell
$$

where $k_{g}$ is the geodesic curvature in $R$.

Let $R_{i}$ be a compact surface with boundary such that $T_{i}=R_{i} \cup S_{i}$ is a complete embedded simply connected surface. Combining again (1) and (2), gives

$$
\int_{R_{i}} K d R_{i}=2 \pi-\int_{\partial R_{i}} k_{g}(s) d s+\frac{1}{\pi} \int_{\mathcal{L}} \#\left(\ell \cap R_{i}\right) d \ell
$$

Applying Theorem 1 to each $T_{i}$, and comparing with (5) yields

$$
\begin{array}{r}
\int_{S_{i}} K d S_{i}=-2 \pi+\frac{1}{\pi} \int_{\mathcal{L}}\left(\#\left(\ell \cap S_{i}\right)-\lambda^{2}\left(\ell, C_{i}\right)\right) d \ell \\
-\frac{1}{\pi} \int_{C_{i} \times C_{i}} \theta \sin \theta \frac{d x d y}{\|y-x\|^{2}}+\int_{\partial R_{i}} k_{g}(s) d s
\end{array}
$$

Addition of (44) and (6) finishes the proof.

1.1. The ideal defect. The last term in (3), which we call the ideal defect, can also be described as an integral in the space of point pairs of $\partial_{\infty} \mathbb{H}^{3} \equiv \mathbb{R}^{2}$, with respect to the Möbius invariant measure on this space.

Proposition 3. Let $\Omega \subset \mathbb{R}^{2}$ be a compact domain bounded by a simple closed curve $C$ of class $\mathcal{C}^{2}$. Then

$$
\int_{C \times C} \theta \sin \theta \frac{d x d y}{\|y-x\|^{2}}=4 \int_{N T(\Omega)} \frac{d z d w}{\|z-w\|^{4}}
$$

where $N T(\Omega) \subset \Omega \times \Omega$ is the set of point pairs $(z, w)$ such that any circle $\xi \subset \mathbb{R}^{2}$ containing $z$ and $w$ intersects $\mathbb{R}^{2} \backslash \Omega$ (i.e. $z, w \in \xi \Rightarrow \xi \not \subset \Omega$.)

Proof. Let $Q \subset \mathbb{H}^{3}$ be the convex hull of $\Omega^{c}=\partial_{\infty} \mathbb{H}^{3} \backslash \Omega$; i.e. $Q$ is the minimal convex set containing $\Omega^{c}$. Using the Klein model, $Q$ can be seen as the euclidean convex hull of $\Omega^{c}$. Let us consider the boundary $S=\partial Q \subset \mathbb{H}^{3}$, which is a surface of class $\mathcal{C}^{1}$. Next we construct a sequence of convex sets $Q_{n} \subset \mathbb{H}^{3}$ such that: $Q_{n} \supset Q_{n+1}, Q=\cap_{n=1}^{\infty} Q_{n}$, and $S_{n}=\partial Q_{n}$ is a $\mathcal{C}^{2}$ surface with cone-like end $C$. First, let $X \in \mathfrak{X}\left(\mathbb{R}^{3}\right)$ be a vector field in the Klein model such that $X$ vanishes only at $C$, and $\left.X\right|_{\Omega}$ points to the interior of the model. Then, for small $t>0$, the flow $\varphi_{t}$ brings $\Omega$ to a surface $\varphi_{t}(\Omega)$ with a cone-like end on $C$, and bounding a convex domain $D$. On the other hand, let $Q$ be approximated by a decreasing sequence $Q_{n}^{\prime} \subset \mathbb{R}^{3}$ of euclidean convex sets with boundary of class $\mathcal{C}^{2}$ (cf. [11]). Then, smoothening the corners of $D \cap Q_{n}^{\prime}$ yields the desired sequence. 
By Theorem 1

$$
\int_{S_{n}} K d S_{n}=\frac{1}{\pi} \int_{\mathcal{L}}\left(\#\left(\ell \cap S_{n}\right)-\lambda^{2}(\ell, C)\right) d \ell-\frac{1}{\pi} \int_{C \times C} \theta \sin \theta \frac{d x d y}{\|y-x\|^{2}} .
$$

Using, for instance, the arguments in [6], one can show

$$
\lim _{n} \int_{S_{n}} K d S_{n}=0
$$

On the other hand, by monotone convergence,

$$
\lim _{n} \int_{\mathcal{L}}\left(\#\left(\ell \cap S_{n}\right)-\lambda^{2}(\ell, C)\right) d \ell=\int_{\mathcal{L}}\left(\#(\ell \cap S)-\lambda^{2}(\ell, C)\right) d \ell .
$$

Hence,

$$
\int_{C \times C} \theta \sin \theta \frac{d x d y}{\|y-x\|^{2}}=\int_{\mathcal{L}}\left(\#(\ell \cap S)-\lambda^{2}(\ell, C)\right) d \ell .
$$

The right hand side above is the measure of geodesics intersecting $Q$ but not $\Omega$. We determine each geodesic $\ell \in \mathcal{L}$ by its ideal endpoints $(z, w)$. This allows to express $d \ell$ as in (16). Finally, we just need to note that a geodesic $\ell$ intersects the convex hull $Q$ if and only if every geodesic 2 -plane containing $\ell$ intersects $\Omega$.

1.2. Integral of the inverse of the chord. Next we express the ideal defect in an alternative way which is not invariant, but still interesting. Let $C \subset \partial_{\infty} \mathbb{H}^{3}$ be a $\mathcal{C}^{2}$-differentiable simple closed curve, and consider $S=C \times(0, \infty) \subset \mathbb{H}^{3}$. We may think of $S$ as a surface with one end by closing the top end at infinity with an infinitesimally small surface. Then, the total curvature of $S$ equals $2 \pi$, and Theorem 1 applied to $S$ yields

$$
\begin{gathered}
2 \pi+\frac{1}{\pi} \int_{C \times C} \theta \sin \theta \frac{d x d y}{\|y-x\|^{2}}=\frac{2}{\pi} \int_{\mathbb{R}^{2} \times \mathbb{R}^{2}}\left(\#(\overline{z w} \cap C)-\lambda^{2}(z, w ; C)\right) \frac{d z d w}{\|w-z\|^{4}} \\
=\frac{2}{\pi} \int_{A(2,1)} \sum_{x, y \in L \cap C} \frac{(-1)^{\#(\overline{x y} \cap C)}}{\|y-x\|} d L
\end{gathered}
$$

where $\overline{z w}$ denotes the line segment joining $z, w \in \mathbb{R}^{2}$, and $d L$ is the invariant measure on the space $A(2,1)$ of (unoriented) lines of $\mathbb{R}^{2}$, normalized as in [10]. The first equality uses (16). The second equality is Proposition 10.

As a consequence, the integral in (7) is invariant under Möbius transformations, which was a priori not obvious. In fact, if $C$ bounds a convex domain $\Omega$, then (7) is

$$
\frac{4}{\pi} \int_{A(2,1)} \frac{1}{\sigma(L \cap \Omega)} d L
$$


where $\sigma(L \cap \Omega)$ is the chord length. The previous functional (8) is one of the so-called Franklin invariants of convex sets, defined by Santaló in [9] as a generalization of a functional introduced by Franklin with motivations from stereology (cf. [4]). These functionals had the nice property of being invariant by dilatations. For instance, the integral (8) could in principle be used to estimate, by means of line sections, the number of particles in a plane region, if these particles have the same shape but possibly different size.

An immediate consequence of our results is that (8) is in fact invariant under the Möbius group. An interesting question is to determine which of the Franklin functionals enjoy this bigger invariance. Besides, it was conjectured that the Franklin invariants are minimal for balls (cf. [4] and [9]). This was shown by Franklin among ellipsoids while Santaló obtained some general non-sharp inequalities. As a consequence of our results, we can prove this conjecture in the planar case.

Corollary 4. For a convex set $\Omega \subset \mathbb{R}^{2}$ we have

$$
\int_{A(2,1)} \frac{1}{\sigma(L \cap \Omega)} d L \geq \frac{\pi^{2}}{2}
$$

where $\sigma$ is the length of the chord, and $A(2,1)$ is the space of lines. Equality holds in (9) if and only if $\Omega$ is a round disk. Moreover, the left hand side of (9) is invariant by Möbius transformations (keeping $\Omega$ convex).

Proof. By (7) we have

$$
\frac{4}{\pi} \int_{A(2,1)} \frac{1}{\sigma(L \cap \Omega)} d L=2 \pi+\frac{1}{\pi} \int_{C \times C} \theta \sin \theta \frac{d x d y}{\|y-x\|^{2}} \geq 2 \pi,
$$

and the equality occurs if and only if $\theta \equiv 0$. Indeed, since $C$ is convex it is easy to see that $-\pi<\theta<\pi$.

The author wishes to thank W.Kühnel, R.Langevin, J.O'Hara and E.Teufel for valuable discussions during the preparation of this work. It is a pleasure to thank the anonymous referee for useful suggestions that have led to a substantial simplification of the paper.

\section{The SPACE OF GeOdesics}

Let $\mathcal{F}=\left\{\left(x ; g_{1}, g_{2}, g_{3}\right)\right\}$ be the bundle of positive orthonormal frames of $\mathbb{H}^{3}$; i.e., each $\left(g_{i}\right)_{i=1,2,3}$ is a positive orthonormal basis of $T_{x} \mathbb{H}^{3}$. We consider on $\mathcal{F}$ the dual and connection forms

$$
\omega_{i}=\left\langle d x, g_{i}\right\rangle, \quad \omega_{i j}=\left\langle\nabla g_{i}, g_{j}\right\rangle
$$

where $\langle$,$\rangle denotes the (hyperbolic) metric in \mathbb{H}^{3}$, and $\nabla$ is the corresponding riemannian connection. The structure equations read

$$
d \omega_{i}=\omega_{j} \wedge \omega_{j i}, \quad d \omega_{i j}=\omega_{i} \wedge \omega_{j}+\omega_{i k} \wedge \omega_{k j}
$$


Let $\mathcal{L}^{+}$be the space of oriented geodesics of $\mathbb{H}^{3}$. Clearly $\mathcal{L}^{+}$is a double cover of $\mathcal{L}$. Consider $\pi_{1}: \mathcal{F} \rightarrow \mathcal{L}^{+}$given by $\pi_{1}\left(x ; g_{1}, g_{2}, g_{3}\right)=\ell$ with $x \in \ell$, and $g_{1} \in T_{x} \ell$ pointing in the positive direction. The space $\mathcal{L}^{+}$can be endowed with a differentiable structure such that $\pi_{1}$ is a smooth submersion. Moreover, $\mathcal{L}^{+}$ admits a volume form $d \ell$ invariant under isometries of $\mathbb{H}^{3}$, which is unique up to normalization, and characterized by (cf.[10])

$$
\pi_{1}^{*}(d \ell)=\omega_{2} \wedge \omega_{12} \wedge \omega_{3} \wedge \omega_{13}
$$

Similarly, one can consider $\mathcal{L}_{2}$, the space of (unoriented) totally geodesic surfaces (geodesic planes) of $\mathbb{H}^{3}$. We will use the space of flags

$$
\mathcal{L}_{1,2}=\left\{(\ell, \wp) \in \mathcal{L}^{+} \times \mathcal{L}_{2} \mid \ell \subset \wp\right\},
$$

and the canonical projection $\pi: \mathcal{L}_{1,2} \rightarrow \mathcal{L}^{+}$which makes $\mathcal{L}_{1,2}$ a principal $\mathbb{S}^{1}$ bundle over $\mathcal{L}^{+}$. Let us project $\pi_{1,2}: \mathcal{F} \rightarrow \mathcal{L}_{1,2}$ so that $\pi_{1,2}\left(x ; g_{i}\right)=(\ell, \wp)$ with $\wp \supset \ell=\pi_{1}\left(x ; g_{i}\right)$ and $g_{3} \perp T_{x} \wp$. Then $\omega_{23}=\pi_{1,2}^{*} \varphi$ for a certain form $\varphi \in \Omega^{1}\left(\mathcal{L}_{1,2}\right)$, which is an invariant global angular form (or connection) of the bundle $\pi$.

Proposition 5. There exists a unique 2-form $\alpha \in \Omega^{2}\left(\mathcal{L}^{+}\right)$such that

$$
\pi^{*}(\alpha)=d \varphi \in \Omega^{2}\left(\mathcal{L}_{1,2}\right) .
$$

where $\varphi$ is the global angular form of $\pi$. Moreover $\alpha \wedge \alpha=2 d \ell$, so that $\alpha$ is an invariant symplectic form on $\mathcal{L}^{+}$.

Proof. Assuming $\alpha$ exists, structure equations (10) give

$$
\pi_{1}^{*}(\alpha)=d \omega_{23}=\omega_{2} \wedge \omega_{3}-\omega_{12} \wedge \omega_{13},
$$

whence

$$
\pi_{1}^{*}(\alpha \wedge \alpha)=-2 \omega_{2} \wedge \omega_{3} \wedge \omega_{12} \wedge \omega_{13}=2 \pi_{1}^{*}(d \ell) .
$$

Therefore $\alpha \wedge \alpha=2 d \ell$ (as $d \pi_{1}$ is exhaustive).

Let $X \in \mathfrak{X}\left(\mathcal{L}_{1,2}\right)$ be the tangent vector field along the fibers of $\pi$ such that $\varphi(X)=1$. By (12), for any $\widetilde{X} \in \mathfrak{X}(\mathcal{F})$ such that $d \pi_{1,2} \widetilde{X}=X$,

$$
\pi_{1,2}^{*}\left(i_{X} d \varphi\right)=i_{\widetilde{X}} d \omega_{23}=0,
$$

whence $i_{X} d \varphi=0$. Then $L_{X} \varphi=0$, and

$$
L_{X} d \varphi=d L_{X} \varphi=0 .
$$

Hence, $d \varphi$ is constant along the fibers of $\pi$, and null on their tangent vectors, which shows the existence of $\alpha$. The uniqueness follows from the injectivity of $\pi^{*}$.

It follows from the previous proposition that

$$
d\left(\pi^{*} \alpha \wedge \varphi\right)=2 \cdot \pi^{*}(d \ell)
$$

This will be used in Section 4 to prove Theorem 1 by means of Stokes' theorem. 
Remark 2. The forms $\varphi, \alpha$ are in some sense dual to the forms $\omega_{1}, d I$ used in [8]. In fact, many of the subsequent constructions are parallel to those of [8]. However, choosing $\alpha$ leads us to results involving the total curvature, while $d I$ made the area appear. This choice could not be done in the euclidean setting since there $\alpha \wedge \alpha$ vanishes.

The following notation will be used throughout the paper:

$$
A \ltimes B:=\{(x, y) \in A \times B \mid x \neq y\} .
$$

In the Poincaré model, by considering the ideal endpoints $z, w$ of each geodesic $\ell$, one identifies (a full-measure subset of) $\mathcal{L}^{+}$with $\mathbb{R}^{2} \ltimes \mathbb{R}^{2}$. Then, an elementary computation with moving frames (cf.(17)) gives the following expression for $\alpha$ at a point $(z, w) \equiv \ell \in \mathcal{L}^{+}$:

$$
\alpha=\frac{2}{\|w-z\|^{2}}\left(d z_{1} \wedge d w_{2}+d z_{2} \wedge d w_{1}\right)
$$

where the coordinate system of $\mathbb{R}^{2}$ has been chosen in such a way that $z_{2}=w_{2}=0$ and $w_{1}=-z_{1}$. In particular, if $z=z(x)$ and $w=w(y)$ are curves parametrized by arc-length, then

$$
\alpha=2 \sin \theta(x, y) \frac{d x \wedge d y}{\|w(y)-z(x)\|^{2}}
$$

where $\theta(x, y)$ is the oriented angle between the two oriented circles through $z(x), w(y)$, tangent to $z^{\prime}(x)$ and $w^{\prime}(y)$ respectively.

Using (14) we can also obtain an expression for the measure of geodesics. Indeed,

$$
d \ell=\frac{1}{2} \alpha \wedge \alpha=4 \frac{d z \wedge d w}{\|w-z\|^{4}}
$$

where $d z, d w$ denote the area elements of the ideal endpoints $z, w$ in $\mathbb{R}^{2}$.

Remark 3. The following complex valued two form in $\mathbb{C} \ltimes \mathbb{C}$ was introduced by Langevin and O'Hara in [5] under the name infinitesimal cross-ratio

$$
\omega_{c r}=\frac{d\left(z_{1}+i z_{2}\right) \wedge d\left(w_{1}+i w_{2}\right)}{(w-z)^{2}}, \quad(z, w) \in \mathbb{C} \ltimes \mathbb{C} .
$$

This form $\omega_{c r}$ is invariant under the diagonal action of the Möbius group $\operatorname{Sl}(2, \mathbb{C})$. Using this fact, one checks easily that $-\alpha / 2$ coincides with $\mathfrak{I m}\left(\omega_{c r}\right)$, the imaginary part of the infinitesimal cross-ratio.

We end the section by showing that the measure of non-trivial geodesics is a natural quantity. This fact was already noticed in the euclidean setting by Pohl (cf. [8], equation (6.5)). 
Proposition 6. Let $S \subset \mathbb{H}^{3}$ be an embedded surface with cone-like ends $C \subset$ $\partial_{\infty} \mathbb{H}^{3}$. Let $\Phi: S \ltimes S \rightarrow \mathcal{L}^{+}$be such that $\Phi(x, y)$ is the oriented geodesic going first through $x$ and then through $y$. Then

$$
\int_{S \ltimes S} \Phi^{*}(d \ell)=\frac{1}{2} \int_{\mathcal{L}^{+}}\left(\#(\ell \cap S)-\lambda^{2}(\ell, C)\right) d \ell .
$$

Proof. By the coarea formula

$$
\int_{S \ltimes S} \Phi^{*}(d \ell)=\int_{\mathcal{L}^{+}} \mu(\ell) d \ell
$$

where

$$
\mu(\ell)=\sum_{(x, y) \in \Phi^{-1}(\ell)}-\epsilon(x) \epsilon(y)
$$

being $\epsilon(u)$ the sign at $u$ of the algebraic intersection $\ell \cdot S$. Now, let $p$ (resp. $q$ ) be the number of points of $\ell \cap S$ with $\epsilon=1$ (resp. $\epsilon=-1$ ), so that

$$
\#(\ell \cap S)=p+q, \quad \lambda(\ell, C)=\ell \cdot S=p-q .
$$

Then $\Phi^{-1}(\ell)$ contains $(p(p-1)+q(q-1)) / 2$ pairs $(x, y)$ with $\epsilon(x)=\epsilon(y)$, and $p q$ elements with $\epsilon(x)=-\epsilon(y)$. Therefore $2 \mu(\ell)=2 p q-p(p-1)-q(q-1)=$ $\#(\ell \cap S)-\lambda^{2}(\ell, C)$.

\section{Convergence Results}

Next we establish the convergence of the integrals appearing in Theorem 1. In the whole section, $S \subset \mathbb{H}^{3}$ will denote a complete surface with a connected cone-like end $C \subset \partial_{\infty} \mathbb{H}^{3}$. Here $\mathbb{H}^{3}$ denotes the Poincaré half-space model. For $h>0$, we set $S_{h}=\left\{x \in S \mid x_{3} \geq h\right\}$ which is a compact surface with boundary $C_{h}=\partial S_{h}=\left\{x \in S \mid x_{3}=h\right\}$.

Proposition 7. If $K$ denotes the extrinsic curvature of $S$, and $d S$ is the area element, then

$$
\int_{S} K d S
$$

is absolutely convergent.

Proof. Let us consider the global orthonormal frame $e_{i}(x)=x_{3} \partial / \partial x_{i},(i=1,2,3)$ defined for all $x \in \mathbb{H}^{3}$. The connection forms $\theta_{i j}=\left\langle\nabla e_{i}, e_{j}\right\rangle$ are then given by

$$
\theta_{i 3}=\frac{d x_{i}}{x_{3}}, \quad \theta_{i j}=0 \quad \text { for } i, j \neq 3
$$

Let us fix now $y \in S$. After a change of coordinates, we can assume $e_{2}(y) \in T_{y} S$. Let $v_{1}, v_{2}, v_{3}$ be a frame locally defined on $S$ (around $y$ ) so that $v_{2}(y)=e_{2}(y)$, and $v_{1}(x), v_{2}(x) \in T_{x} S$. Then $v_{i}(x)=a_{i j}(x) e_{j}(x)$ for an orthogonal matrix $\left(a_{i j}(x)\right) \in$ 
$\mathrm{O}(3)$. In particular $v_{1}(y)=\cos \alpha e_{1}+\sin \alpha e_{3}$, and $v_{3}(y)=-\sin \alpha e_{1}+\cos \alpha e_{3}$ for some $\alpha \in[0,2 \pi)$. Then $\left(\omega_{i j}\right)_{y}=\left\langle\nabla v_{i}, v_{j}\right\rangle_{y}$ are given by

$$
\begin{gathered}
\left(\omega_{12}\right)_{y}=\left\langle\nabla\left(a_{1 i} e_{i}\right), e_{2}\right\rangle_{y}=d a_{12}+a_{12}(y)\left\langle\nabla e_{1}, e_{2}\right\rangle+a_{32}(y)\left\langle\nabla e_{3}, e_{2}\right\rangle \\
=d a_{12}+\cos \alpha \theta_{12}+\sin \alpha \theta_{32} \stackrel{\sqrt{17})}{=} d a_{12}-\sin \alpha \frac{d x_{2}}{y_{3}} \\
\left(\omega_{13}\right)_{y}=\left\langle\nabla\left(a_{1 i} e_{i}\right),-\sin \alpha e_{1}+\cos \alpha e_{3}\right\rangle_{y} \\
=-\sin \alpha\left(d a_{11}+a_{1 i}(y) \theta_{i 1}\right)+\cos \alpha\left(d a_{13}+a_{1 i}(y) \theta_{i 3}\right) \\
=-\sin \alpha d a_{11}+\cos \alpha d a_{13}+\frac{d x_{1}}{y_{3}}
\end{gathered}
$$

and similarly

$$
\left(\omega_{23}\right)_{y}=-\sin \alpha d a_{21}+\cos \alpha d a_{23}+\cos \alpha \frac{d x_{2}}{y_{3}}
$$

In particular

$$
\omega_{13}\left(v_{1}\right)=-\sin \alpha d a_{11}\left(v_{1}\right)+\cos \alpha d a_{13}\left(v_{1}\right)+\frac{d x_{1}\left(v_{1}\right)}{y_{3}}=O\left(y_{3}\right)+\frac{d x_{1}\left(v_{1}\right)}{y_{3}} .
$$

But $d x_{1}\left(v_{1} / y_{3}\right)=\cos \alpha=O\left(y_{3}\right)$. Indeed, $\cos \alpha=\left\langle e_{1}, v_{1}\right\rangle$ is a $\mathcal{C}^{1}$ function on $\bar{S}=S \cup C$ and vanishes at $C$. One checks similarly that $\omega_{i 3}\left(v_{j}\right)=O\left(y_{3}\right)$ for $i, j=1,2$. We have thus that

$$
K(y)=\operatorname{det}\left(\omega_{i 3}\left(v_{j}\right) \mid i, j=1,2\right)=\omega_{13}\left(v_{1}\right) \omega_{23}\left(v_{2}\right)-\omega_{13}\left(v_{2}\right) \omega_{23}\left(v_{1}\right)=O\left(y_{3}^{2}\right)
$$

The result follows since $y_{3}^{2} d S$ is the euclidean area element of $S$ in the model.

The following proposition is a first step towards the existence of formula (3).

Proposition 8. Let $S, R \subset \mathbb{H}^{3}$ be two surfaces with the same cone-like end $\partial_{\infty} S=$ $\partial_{\infty} R \subset \partial_{\infty} \mathbb{H}^{3}$. Then

$$
\int_{S} K d S-\int_{R} K d R=2 \pi(\chi(S)-\chi(R))+\lim _{h \rightarrow 0} \frac{1}{\pi} \int_{\mathcal{L}}\left(\#\left(\ell \cap S_{h}\right)-\#\left(\ell \cap R_{h}\right)\right) d \ell .
$$

Proof. From (11) and (2) one gets

$$
\int_{S_{h}} K d S_{h}=2 \pi \chi\left(S_{h}\right)+\frac{1}{\pi} \int_{\mathcal{L}} \#\left(\ell \cap S_{h}\right) d \ell-\int_{\partial S_{h}} k_{g}(s) d s
$$

and similarly for $R_{h}$. We must show that

$$
\int_{\partial S_{h}} k_{g}(s) d s-\int_{\partial R_{h}} k_{g}(s) d s
$$

tends to zero as $h \rightarrow 0$. By equation (18) we have

$$
k_{g}=-\omega_{12}\left(v_{2}\right)=-d a_{12}\left(v_{2}\right)+\sin \alpha .
$$


In the previous proof we learned that $\cos \alpha=O(h)$, and thus $\sin \alpha=1+O\left(h^{2}\right)$. Besides, in the choice of the local frame $v_{1}, v_{2}, v_{3}$ one could further assume that $v_{1}$ is everywhere orthogonal to $e_{2}$. Hence $a_{12}=\left\langle v_{1}, e_{2}\right\rangle \equiv 0$, so $k_{g}=\sin \alpha=1+O\left(h^{2}\right)$, and

$$
\int_{\partial S_{h}}\left(k_{g}(s)-1\right) d s=\int_{\partial S_{h}} O\left(h^{2}\right) d s=O(h),
$$

and similarly for $\partial R_{h}$. Thus, it suffices to show that the difference of (hyperbolic) lengths of $\partial S_{h}$ and $\partial R_{h}$ tends to zero as $h \rightarrow 0$. This follows from the fact that $\partial_{\infty} S$ is an euclidean geodesic of both $S$ and $R$, and geodesics are extremals of the length. Indeed, the euclidean lengths of $\partial S_{h}$ and $\partial R_{h}$ differ both from the length of $\partial_{\infty} S$ with an order $O\left(h^{2}\right)$. Hence, their respective hyperbolic lengths have a difference of order $O(h)$.

Next we study the convergence of the measure of non-trivial geodesics.

Lemma 9. If $\lambda\left(\ell, C_{h}\right)$ denotes the linking number (defined up to sign) of a geodesic $\ell$ with the curve $C_{h}$, then

$$
\lim _{h \rightarrow 0} \int_{\mathcal{L}}\left(\#\left(\ell \cap S_{h}\right)-\lambda^{2}\left(\ell, C_{h}\right)\right) d \ell=\int_{\mathcal{L}}\left(\#(\ell \cap S)-\lambda^{2}(\ell, C)\right) d \ell
$$

where $\lambda(\ell, C)$ is the limit of $\lambda\left(\ell, C_{h}\right)$ when $h \rightarrow 0$.

Proof. Let $\ell \in \mathcal{L}$ be transverse to $S$, which happens for almost every $\ell$. Then $\#\left(\ell \cap S_{h}\right)$ is an increasing function of $h$. For $h$ small enough, $C_{h}$ is connected, and thus $\lambda^{2}\left(\ell, C_{h}\right) \leq 1$. Therefore $\#\left(\ell \cap S_{h}\right)-\lambda^{2}\left(\ell, C_{h}\right)$ is an increasing function of $h$. Then (20) follows by monotone convergence.

We will see below, that the limit in (20) is finite. For the moment, we show this fact for the infinite cylinder over $C$.

Proposition 10. Let $C \subset \partial_{\infty} \mathbb{H}^{3}$ be a simple closed curve, and let $R=C \times(0, \infty) \subset$ $\mathbb{H}^{3}$. Then the following integrals converge and coincide

$$
\int_{\mathcal{L}}\left(\#(\ell \cap R)-\lambda^{2}(\ell, C)\right) d \ell=\int_{A(2,1)} \sum_{x, y \in L \cap C} \frac{(-1)^{\#(\overline{x y} \cap C)}}{\|y-x\|} d L<\infty
$$

where $d L$ is an invariant measure in the space $A(2,1)$ of lines in $\mathbb{R}^{2}$.

Proof. After a vertical projection onto $\partial_{\infty} \mathbb{H}^{3}$, each geodesic $\ell$ is mapped to a segment $\overline{z w}$, and $R$ projects onto $C$. From the proof of Proposition 6 we know

$$
\#(\ell \cap R)-\lambda^{2}(\ell, C)=-\sum_{x, y \in \overline{z w} \cap C} \epsilon(x) \epsilon(y)
$$

where $\epsilon(u)$ is the sign at $u$ of the algebraic intersection $\overline{z w} \cdot C$. The equality of the integrals follows from (16), together with (cf.[10], equation (4.2))

$$
d z d w=\|t-s\| d s d t d L
$$


where $s, t$ are arc-length parameters of $z, w$ along $L$. In order to check the convergence, we use the following expression of the measure of lines in $\mathbb{R}^{2}$ (cf. [8])

$$
d L=\left|\sin \beta_{x} \sin \beta_{y}\right| \frac{d x d y}{\|y-x\|}
$$

where $x, y$ are intersection points with $C$, and $\beta_{x}, \beta_{y}$ are the oriented angles between $L$ and $C$ at $x, y$ respectively. Then the integral over $A(2,1)$ above becomes

$$
-\int_{C \times C} \sin \beta_{x} \sin \beta_{y} \frac{d x d y}{\|y-x\|^{2}} .
$$

This integral converges since $\beta_{x}, \beta_{y}=O(\|y-x\|)$ as one can easily prove.

Lemma 11. Let $S, R \subset \mathbb{H}^{3}$ be two surfaces with the same cone-like end $\partial_{\infty} S=$ $\partial_{\infty} R \subset \partial_{\infty} \mathbb{H}^{3}$. Then the following integrals are uniformly bounded for all $h>0$

$$
\int_{\mathcal{L}}\left(\lambda^{2}\left(\ell, \partial S_{h}\right)-\lambda^{2}\left(\ell, \partial R_{h}\right)\right) d \ell
$$

Proof. Let $T_{h}$ be the region of $\left\{x \in \mathbb{H}^{3} \mid x_{3}=h\right\}$ bounded by $\partial S_{h}$ and $\partial R_{h}$. If a geodesic $\ell$ is disjoint from $T_{h}$, then $\lambda^{2}\left(\ell, \partial S_{h}\right)=\lambda^{2}\left(\ell, \partial T_{h}\right)$. Hence, the integral above is bounded by the measure of geodesics intersecting $T_{h}$. By the Crofton formula (2), this measure is proportional to the area of $T_{h}$. Since $S$ and $R$ are tangent at infinity, the euclidean area of $T_{h}$ has order $O\left(h^{2}\right)$. Therefore, its hyperbolic area is uniformly bounded.

Proposition 12. The measure of non-trivial geodesics

$$
\int_{\mathcal{L}}\left(\#(\ell \cap S)-\lambda^{2}(\ell, C)\right) d \ell
$$

is absolutely convergent.

Proof. Clearly

$$
\begin{aligned}
\int_{\mathcal{L}}\left(\#\left(\ell \cap S_{h}\right)\right. & \left.-\lambda^{2}\left(\ell, \partial S_{h}\right)\right) d \ell=\int_{\mathcal{L}}\left(\#\left(\ell \cap S_{h}\right)-\#\left(\ell \cap R_{h}\right)\right) d \ell \\
& +\int_{\mathcal{L}}\left(\#\left(\ell \cap R_{h}\right)-\lambda^{2}\left(\ell, \partial R_{h}\right)\right) d \ell+\int_{\mathcal{L}}\left(\lambda^{2}\left(\ell, \partial R_{h}\right)-\lambda^{2}\left(\ell, \partial S_{h}\right)\right) d \ell .
\end{aligned}
$$

The last three integrals are uniformly bounded by Propositions [8, and 10] and Lemma 11 respectively. Thus, by monotonicity, the following limit

$$
\lim _{h \rightarrow 0} \int_{\mathcal{L}}\left(\#\left(\ell \cap S_{h}\right)-\lambda^{2}\left(\ell, \partial S_{h}\right)\right) d \ell
$$

exists and is finite. Since $\#(\ell \cap S)-\lambda^{2}(\ell, C)$ is positive, Lemma 9 shows the absolute convergence of the integral. 
Corollary 13. Let $S \subset \mathbb{H}^{3}$ be a surface with a cone-like end $C \subset \partial_{\infty} \mathbb{H}^{3}$. Then

$$
\int_{S} K d S=2 \pi \chi(S)+\frac{1}{\pi} \int_{\mathcal{L}}\left(\#(\ell \cap S)-\lambda^{2}(\ell, C)\right) d \ell-\delta(C)
$$

where $\delta(C)$ depends only on the ideal curve $C$. All the integrals above are absolutely convergent.

Proof. The convergence has been established in Propositions 7 and 12, The result follows then from Proposition 8 .

Remark 4. We have assumed $C$ to be connected for simplicity. If $C$ is a collection of disjoint simple closed curves, each of them arbitrarily oriented, the previous results hold without change. The key fact for the convergence is that $\lambda^{2}(\cdot, C) \leq 1$ outside a compact subset of $\mathcal{L}$. As for $\delta(C)$, it depends in this case on the orientations of $C$, as well as the relative positions of the several components.

Remark 5. In order to get explicit expressions of $\delta(C)$, it is enough to find, for each curve $C \subset \partial_{\infty} \mathbb{H}^{3}$, a surface $S$ with cone-like ends on $C$ for which the total curvature and the measure of non-trivial geodesics can be computed. In fact, this is what we did in subsections 1.1 and 1.2 .

However, in order to get the expression of $\delta(C)$ that appears in Theorem 11, we will need to follow a different strategy.

\section{Proof of Theorem 1}

4.1. The space of chords. Given a $\mathcal{C}^{2}$-differentiable manifold $S$ (without boundary), the space of chords of $S$ is a $\mathcal{C}^{1}$-differentiable manifold $M_{S}$ with boundary, introduced by Whitney in [14, and described in detail in [8]. This space is the blow-up of $S \times S$ along the diagonal. In particular, the interior of $M_{S}$ is $S \ltimes S$, and the boundary is the sphere bundle of oriented tangent directions of $S$

$$
\partial M_{S}=T^{+} S:=(T S \backslash\{(x, \overrightarrow{0}) \mid x \in S\}) / \mathbb{R}^{+} .
$$

The reader is referred to [8] for details on the differentiable structure of $M_{S}$. The following property describes this structure quite well: given a regular injective $\mathcal{C}^{2}$-differentiable curve $x:[0,1) \rightarrow S$, the curve $c:(0,1) \rightarrow S \ltimes S$ defined by $c(t)=(x(0), x(t))$ extends to a $\mathcal{C}^{1}$-differentiable curve $c:[0,1) \rightarrow M_{S}$ which meets $\partial M_{S}$ transversely at $c(0)=\left[x^{\prime}(0)\right] \in T^{+} S$. Another basic property is the following: the natural projections $p_{1}, p_{2}: S \ltimes S \rightarrow S$ extend naturally to differentiable submersions $p_{1}, p_{2}: M_{S} \rightarrow S$.

Let now $S$ be a manifold with boundary. The space $M_{S}$ of chords of $S$ is constructed as follows. We consider a manifold without boundary $\tilde{S}$ extending $S$. Let $p_{1}, p_{2}: M_{\tilde{S}} \rightarrow \tilde{S}$ be the submersions mentioned above. The space of chords of $S$ is then defined as $M_{S}=p_{1}^{-1}(S) \cap p_{2}^{-1}(S) \subset M_{\tilde{S}}$ (i.e. $M_{S}$ contains the chords of $\tilde{S}$ with both ends in $S$ ). This space is a topological manifold with boundary, 
but this boundary is not smooth. Indeed, the interior of $M_{S}$ is $S \ltimes S$, and the boundary is $\partial M_{S}=T^{+} S \cup(S \ltimes \partial S) \cup(\partial S \ltimes S)$. The faces $T^{+} S, S \ltimes \partial S$, and $\partial S \ltimes S$ are pairwise transverse outside $T^{+} \partial S$ (in fact, $\partial S \ltimes S$ and $S \ltimes \partial S$ are tangent at points of $T^{+} \partial S$ ). Hence, $M_{S} \backslash T^{+} \partial S$ is a manifold with corners in the usual sense (cf. for instance [7]).

4.2. Bundles and sections. In this subsection we use the Klein model of hyperbolic space. Hence $\mathbb{H}^{3}$ is the interior of the closed unit ball $\mathbb{B}^{3}$ in $\mathbb{R}^{3}$. Let $\Psi: \mathbb{B}^{3} \ltimes \mathbb{B}^{3} \rightarrow \mathcal{L}^{+}$be such that $(x, y)$ is mapped to the geodesic line going first through $x$ and then through $y$. This map extends naturally to $\Psi: M_{\mathbb{B}^{3}} \backslash T^{+} \mathbb{S}^{2} \rightarrow$ $\mathcal{L}^{+}$. This extension is smooth by the results of [8].

Let now $S^{\circ} \subset \mathbb{H}^{3}$ be a simply connected surface with a cone-like end $C \subset \partial_{\infty} \mathbb{H}^{3}$. Then, the closure $S=S^{\circ} \cup C$ is a compact surface with boundary in $\mathbb{B}^{3}$, transverse to the ideal sphere $\mathbb{S}^{2}=\partial_{\infty} \mathbb{H}^{3}$. Notice that we slightly modified, for simplicity, the notation used in the previous sections.

As seen in [8, the inclusion $M_{S} \subset M_{\mathbb{B}^{3}}$ is compatible with the differentiable structures. Hence, the mapping

$$
\Phi: M_{S} \backslash T^{+} C \rightarrow \mathcal{L}^{+}
$$

obtained as a restriction of $\Psi$ is smooth. Note that this extends the mapping $\Phi$ defined in Proposition 6 .

To simplify the notation we denote $B:=M_{S} \backslash T^{+} C$. By Proposition 6 , the measure of non-trival geodesics can be obtained by integrating $\Phi^{*}(d \ell)$ on $B$. Our aim is to compute this integral by means of Stokes' theorem, using an invariant form whose differential is $d \ell$. Such a form is given by (13), but it lives in the bundle $\mathcal{L}_{1,2}$. In fact, there is no invariant form in $\mathcal{L}^{+}$whose differential is $d \ell$. We are thus led to consider the pull-back by $\Phi$ of the $\mathbb{S}^{1}$-bundle $\pi: \mathcal{L}_{1,2} \rightarrow \mathcal{L}^{+}$. More precisely, we consider $E:=\Phi^{*}\left(\mathcal{L}_{1,2}\right)=\left\{(z, \wp) \in B \times \mathcal{L}_{2} \mid \Phi(z) \subset \wp\right\}$, and the following commutative diagram with the obvious mappings

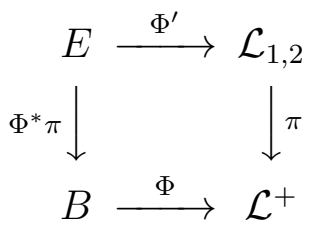

It would be desirable to define a section of $\Phi^{*} \pi: E \rightarrow B$. This section should be canonically constructed in some geometric way. This can be done quite naturally, but only at the boundary $\partial B$; in fact only on

$$
\partial B \backslash(C \ltimes C)=\left(T^{+} S \backslash T^{+} C\right) \cup\left(S^{\circ} \times C\right) \cup\left(C \times S^{\circ}\right)=\partial M_{S} \backslash M_{C} .
$$

Indeed, for $z=(x,[v]) \in T^{+} S \backslash T^{+} C$ we choose the geodesic plane $\wp(z)$ spanned by $T_{x} S$. For $z=(x, y) \in C \times S^{\circ}$, and for $z=(y, x) \in S^{\circ} \times C$, we choose the plane $\wp(z)$ tangent to $C$ at $x$ and containing $y$. Note that this definition does not 
extend to $C \ltimes C$ : the two planes through $x, y \in C$ that are tangent to $C$ at $x$ and $y$ respectively, form a certain angle. In fact, this is precisely the angle $\theta$ appearing in Theorem 1.

To summarize, we have defined

$$
\begin{aligned}
s: \partial M_{S} \backslash M_{C} & \longrightarrow E \\
z & \longmapsto(z, \wp(z)))
\end{aligned}
$$

in such a way that $T_{x} \wp(z)=T_{x} S$ if $z=(x,[v]) \in T^{+} S$, and $T_{x} C \subset T_{x} \wp(z)$ for $z=(x, y) \in S^{\circ} \times C$, or $z=(y, x) \in C \times S^{\circ}$.

We already noted that $s$ has a jump discontinuity in $C \ltimes C$. To solve this, we shall complete the image of $s$ with a family of fiber intervals interpolating the two one-sided limits of $s$. However, these intervals are not well-defined in the $\mathbb{S}^{1}$-bundle $E$. We are led to consider an infinite cyclic cover of $E$ that gives an $\mathbb{R}$-bundle over $B$. Next we define this cover, and we show it admits a lift of $s$. Here we take great advantage of the assumption that $S$ is simply connected.

Proposition 14. The principal $\mathbb{S}^{1}$-bundle $\Phi^{*} \pi: E \longrightarrow B$ is trivial. Moreover, there is a bundle isomorphism $\tau: E \longrightarrow B \times \mathbb{S}^{1}$, such that $\tau \circ s$ lifts over the covering $q: B \times \mathbb{R} \rightarrow B \times \mathbb{S}^{1}$; i.e., there exists a continuous function

$$
g: \partial M_{S} \backslash M_{C} \rightarrow \mathbb{R}
$$

such that $q(x, g(x))=\tau \circ s(x)$ for every $x \in \partial M_{S} \backslash M_{C}$.

Proof. Consider an isotopy of embeddings $H: S \times[0,1] \rightarrow \mathbb{B}^{3}$ such that $H_{0}=i d$ and $H_{1}\left(S^{\circ}\right)$ is contained in a plane $\wp \in \mathcal{L}_{2}$. We may construct the isotopy so that $H(C \times[0,1]) \subset \mathbb{S}^{2}$. Put $\tilde{H}(x, y, t):=\left(H_{t}(x), H_{t}(y)\right)$ for $(x, y) \in S \ltimes S$. Clearly $\tilde{H}$ extends continuously to $\tilde{H}: B \times[0,1] \rightarrow M_{\mathbb{B}^{3}} \backslash T^{+} \mathbb{S}^{2}$. Furthermore the bundle $\left(\Psi \circ \tilde{H}_{1}\right)^{*} \pi$ clearly admits a global section $s_{1} \equiv \wp$. By the covering homotopy theorem, $s_{1}$ extends to a global section $\tilde{s}$ of $(\Psi \circ \tilde{H})^{*} \pi$, and therefore this principal bundle is trivial. This already shows that $E=\left(\Psi \circ \tilde{H}_{0}\right)^{*}\left(\mathcal{L}_{1,2}\right)$ is trivial. Let

$$
\tilde{\tau}:(\Psi \circ \tilde{H})^{*}\left(\mathcal{L}_{1,2}\right) \rightarrow M_{S} \times[0,1] \times \mathbb{S}^{1}
$$

be the isomorphism corresponding to this global section, i.e. such that $\tilde{\tau} \circ \tilde{s}(z, t)=$ $(z, t, 1)$. For each $t$, the construction above (cf.(22)) yields a section $s_{t}$ of the restriction of $\Psi^{*} \pi$ to each $\partial M_{S_{t}} \backslash M_{\partial S_{t}}$, with $s_{1} \equiv \wp$, and $s_{0}=s$. Clearly these fit together to give a global section $\bar{s}$ of the restriction of $(\Psi \circ \tilde{H})^{*} \pi$ to $\partial M_{S} \backslash M_{C} \times[0,1]$. From the construction of $\tilde{\tau}$ it is clear that the restriction of $\tilde{\tau} \circ \bar{s}$ to $\partial M_{S} \backslash M_{C} \times\{1\}$ lifts over $q$. Now the covering homotopy theorem implies that $\tilde{\tau} \circ \bar{s}$ lifts over all of $\partial M_{S} \backslash M_{C} \times[0,1]$. Hence we may take $\tau$ to be the restriction of $\tilde{\tau}$ to $\left(\Psi \circ \tilde{H}_{0}\right)^{*}\left(\mathcal{L}_{1,2}\right)=E$.

While $g$ can not be continuously defined over all $\partial B$, we can consider the continuous extensions of $g$ to $S \ltimes C$ and $C \ltimes S$ respectively. We denote these extensions 
by $g_{1}$ and $g_{2}$ respectively. This way, $\theta(x, y)=g_{2}(x, y)-g_{1}(x, y)$ in the notation of Theorem 1, for every $(x, y) \in C \ltimes C$. Let $T_{1} \subset B \times \mathbb{R}$ be the graph of $g$ over $\partial B \backslash C \ltimes C$, completed with the graphs of $g_{1}$ and $g_{2}$ over $C \ltimes C$. Now we sew in a family of vertical intervals over $C \ltimes C$ interpolating these two one-sided limits. To be precise we consider $T_{2}=C \ltimes C \times[0,1]$ together with the mapping

$$
\begin{aligned}
\sigma: T_{2} & \longrightarrow C \ltimes C \times \mathbb{R} \\
(x, y, t) & \longmapsto\left(x, y, t g_{1}(x, y)+(1-t) g_{2}(x, y)\right)
\end{aligned}
$$

Note that $\sigma$ is a smooth mapping, possibly non-regular.

In the following we will need to specify some orientations. The manifold $S \ltimes S$, and hence $M_{S}$ is canonically oriented by $d S \wedge d S$. This induces an orientation on $\partial M_{S}$, and hence $T_{1}$ is naturally oriented. Finally, we choose on $T_{2}$ the orientation given by $d x \wedge d y \wedge d t$. This way, $T_{1}$ and $T_{2}$ induce opposite orientations on the graphs of $g_{1}$ and $g_{2}$.

4.3. Stokes' theorem. Before applying Stokes' theorem, the non-compacity of $M_{S} \backslash T^{+} C$ needs to be settled. To this end, let us consider the function $f: M_{S} \rightarrow$ $[0, \infty]$ which vanishes on $T^{+} C$, and assigns to each $z \in M_{S} \backslash T^{+} C$ the euclidean distance in $\partial_{\infty} \mathbb{H}^{3}$ between the ideal endpoints of $\Phi(z)$. Here $\mathbb{H}^{3}$ denotes again the Poincaré model. Then

$$
\Delta_{\epsilon}:=f^{-1}([0, \epsilon))
$$

is a neigborhood of $T^{+} C$ inside $M_{S}$, and $M_{S} \backslash \Delta_{\epsilon}$ is compact. By Sard's theorem, for almost every $\epsilon$, the level set $\partial \Delta_{\epsilon}:=f^{-1}(\epsilon)$ is smooth and transverse to $\partial M_{S}$. Therefore $M_{S} \backslash \Delta_{\epsilon}$ is a compact manifold with corners for almost every $\epsilon>0$. We denote this manifold by $B_{\epsilon}:=M_{S} \backslash \Delta_{\epsilon}$.

Let us consider $T_{1, \epsilon}=T_{1} \backslash \Delta_{\epsilon}^{\prime}$, being $\Delta_{\epsilon}^{\prime}=\pi^{-1}\left(\Delta_{\epsilon}\right)$. Here $\pi: B \times \mathbb{R} \rightarrow B$ is the projection on the first factor. For a generic $\epsilon>0$, Sard's theorem applied to $f \circ \pi$ ensures that $T_{1, \epsilon}$ is a compact manifold with corners. Also $T_{2, \epsilon}=T_{2} \backslash \sigma^{-1}\left(\Delta_{\epsilon}^{\prime}\right)$ is a compact manifold with corners for almost every $\epsilon$.

Since $T_{2, \epsilon}$ can be triangulated, we may think of $\left(T_{2, \epsilon}, \sigma\right)$ as a (smooth) singular chain. Also $T_{1, \epsilon}$ can be thought of as a singular chain. Hence it makes sense to consider $T_{\epsilon}:=T_{1, \epsilon}+T_{2, \epsilon}$ as a chain in $\partial B \times \mathbb{R} \backslash \Delta_{\epsilon}^{\prime}$. Its boundary is a singular chain of $\partial \Delta_{\epsilon}^{\prime}:=\pi^{-1} \partial \Delta_{\epsilon}$, namely $\partial T_{\epsilon}=\left(T_{1} \cap \partial \Delta_{\epsilon}^{\prime}\right)+\sigma^{-1}\left(\partial \Delta_{\epsilon}^{\prime}\right)$.

In the next subsection, we will construct a chain $R_{\epsilon}$ in $\partial \Delta_{\epsilon}^{\prime}$ such that $\partial R_{\epsilon}=$ $-\partial T_{\epsilon}$. This way, $T_{\epsilon}+R_{\epsilon}$ is a cycle, and hence gives an element in the homology group $H_{3}\left(B_{\epsilon} \times \mathbb{R}\right)$. Since $S$ is contractible, we have the following homotopy equivalences

$$
B_{\epsilon} \times \mathbb{R} \simeq B_{\epsilon} \simeq B \simeq S \ltimes S \simeq S \times \mathbb{S}^{1} \simeq \mathbb{S}^{1}
$$

Therefore $H_{3}\left(B_{\epsilon} \times \mathbb{R}\right)=0$, and $T_{\epsilon}+R_{\epsilon}$ is a boundary.

By composing with $\pi: B \times \mathbb{R} \rightarrow B$ we can consider $\pi_{*}\left(T_{\epsilon}+R_{\epsilon}\right)$ as a cycle in $(\partial B) \backslash \Delta_{\epsilon} \cup \partial \Delta_{\epsilon}=\partial B_{\epsilon}$. The latter is an oriented compact manifold so $H_{3}\left(\partial B_{\epsilon}, \mathbb{Z}\right) \equiv$ 
$\mathbb{Z}$, and $\left[\pi_{*}\left(T_{\epsilon}+R_{\epsilon}\right)\right]$ is given by some integer $n$. For any form $\omega \in \Omega^{3}\left(\partial B_{\epsilon}\right)$ one has

$$
\int_{T_{\epsilon}+R_{\epsilon}} \pi_{*} \omega=n \int_{\partial B_{\epsilon}} \omega .
$$

Note that $\pi$ restricted to the interior of $T_{1, \epsilon}$ is a diffeomorphism preserving orientations. Thus, taking $\omega$ supported on the interior of $\pi\left(T_{1, \epsilon}\right)$ makes clear that $n=1$.

Now, since $H^{4}\left(B_{\epsilon}\right)=0$, there exists some differential form $\omega \in \Omega^{3}\left(B_{\epsilon}\right)$ such that $d \omega=\Phi^{*} d \ell$. Therefore, by Stokes' theorem

$$
\int_{B_{\epsilon}} \Phi^{*} d \ell=\int_{B_{\epsilon}} d \omega=\int_{\partial B_{\epsilon}} \omega=\int_{T_{\epsilon}+R_{\epsilon}} \pi^{*} \omega=\frac{1}{2} \int_{T_{\epsilon}+R_{\epsilon}} \pi^{*} \alpha \wedge \varphi,
$$

since $2 \pi^{*} \omega-\pi^{*} \alpha \wedge \varphi$ is closed by (13), and $T_{\epsilon}+R_{\epsilon}$ is a boundary. Here we are abusing the notation for simplicity: by $\alpha$ and $\varphi$ we refer to $\Phi^{*} \alpha$ and $\left(\Phi^{\prime} \circ \tau^{-1} \circ \pi\right)^{*} \varphi$ respectively. We will go on with this abuse, and hopefully no confusion will arise.

4.4. Total curvature and ideal defect. In this section we integrate $\pi^{*} \alpha \wedge \varphi$ over $T_{1}$ and $T_{2}$. We will get respectively the total curvature, and the ideal defect.

\section{Proposition 15.}

$$
\lim _{\epsilon \rightarrow 0} \int_{T_{1} \backslash \Delta_{\epsilon}^{\prime}} \pi^{*} \alpha \wedge \varphi=2 \pi \int_{S} K d S
$$

Proof. Recall that

$$
T_{1}=\left(\text { graph }\left.g\right|_{T^{+} S}\right) \cup\left(\text { graph } g_{1}\right) \cup\left(\text { graph } g_{2}\right)
$$

We claim that $\pi^{*} \alpha \wedge \varphi$ vanishes on the graphs of $g_{1}$ and $g_{2}$. Recall these functions are defined over $S \ltimes C$ and $C \ltimes S$ respectively. Indeed, let $x$ be a local coordinate on $C$. Then expression (14) shows $\alpha \wedge d x=0$. Let now $c(t)$ be the lift in the graph of $g$ of a curve $(y(t), x) \in S \times C$ or $(x, y(t)) \in C \times S$ with $x$ fixed. This curve corresponds to a curve $(\ell(t), \wp(t))=\Phi^{\prime} \circ q(c(t)) \in \mathcal{L}_{1,2}$. In the Poincaré model, the ideal boundaries of $\wp(t)$ are circles in $\partial_{\infty} \mathbb{H}^{3}$ tangent to $C$ at the point $x$. In order to compute $\varphi\left(c^{\prime}(t)\right)$ we take an isometry of $\mathbb{H}^{3}$ sending the point $x \in C$ to infinity. This way, $\ell(t)$ become vertical lines, and the geodesic planes $\wp(t)$ are transformed into a family of parallel vertical planes. By using the expression (17) of the connection forms, it is clear that $\varphi\left(c^{\prime}(t)\right)$ vanishes. This shows that $\varphi$ is a multiple of $\pi^{*} d x$ (on this region of $T_{1}$ ), and the claim follows.

We focus now on the graph over $T^{+} S$. Given $(x, l) \in T^{+} S^{\circ}$, we take $v_{1}, v_{2}, v_{3}$ an orthonormal basis of $T_{x} \mathbb{H}^{3}$ such that $\left[v_{1}\right]=l$, and $v_{3} \perp T_{x} S$. With such a moving frame, by (12)

$$
\pi^{*} \alpha \wedge \varphi=\left(\omega_{2} \wedge \omega_{3}-\omega_{12} \wedge \omega_{13}\right) \wedge \omega_{23}=-\omega_{12} \wedge \omega_{13} \wedge \omega_{23}=-K(x) \omega_{12} \wedge d S .
$$


By Proposition [7, this volume form has finite integral on $T^{1} S$, the euclidean unit tangent of $S$ (in the Poincaré model). Then we may use Lebesgue's dominated convergence theorem to get

$$
\begin{aligned}
\lim _{\epsilon \rightarrow 0} \int_{\text {graph }\left.g\right|_{T^{+} S} \backslash \Delta_{\epsilon}^{\prime}} \pi^{*} \alpha \wedge \varphi=\lim _{\epsilon \rightarrow 0} \int_{T^{+} S \backslash \Delta_{\epsilon}} K & \omega_{12} \wedge d S \\
& =\int_{T^{+} S} K \omega_{12} \wedge d S=2 \pi \int_{S} K d S,
\end{aligned}
$$

where we used the natural orientation of $T^{+} S$, which is opposite to the one induced by $M_{S}$.

\section{Proposition 16.}

$$
\lim _{\epsilon \rightarrow 0} \int_{T_{2} \backslash \Delta_{\epsilon}^{\prime}} \pi^{*} \alpha \wedge \varphi=2 \int_{C \times C} \theta \sin \theta \frac{d x d y}{\|y-x\|^{2}} .
$$

Proof. Recall that $T_{2}$ is mapped to the union of vertical segments in $B \times \mathbb{R}$ interpolating the one-sided limits of $g$ along $C \ltimes C$ (cf. (23)). This segments have length $\theta$, and $\varphi$ restricted to the fibers is precisely the length element. Hence, Fubini's theorem gives

$$
\lim _{\epsilon \rightarrow 0} \int_{T_{2} \backslash \Delta_{\epsilon}^{\prime}} \pi^{*} \alpha \wedge \varphi=\lim _{\epsilon \rightarrow 0} \int_{C \times C \backslash \Delta_{\epsilon}} \theta \alpha=2 \lim _{\epsilon \rightarrow 0} \int_{C \times C \backslash \Delta_{\epsilon}} \theta \sin \theta \frac{d x d y}{\|y-x\|^{2}}
$$

where we have used (15). The result follows since $\theta=O(\|y-x\|)$, which is easy to prove.

The proof of Theorem 1 is almost finished. So far we have seen (cf. Propositions 6] and 12, equation (24), and Propositions 15 and 16)

$$
\begin{aligned}
\int_{\mathcal{L}}\left(\#(\ell \cap S)-\lambda^{2}(\ell, C)\right) d \ell & =2 \int_{B} \Phi^{*}(d \ell)=2 \lim _{\epsilon \rightarrow 0} \int_{B_{\epsilon}} \Phi^{*}(d \ell) \\
= & \lim _{\epsilon \rightarrow 0} \int_{R_{\epsilon}} \pi^{*} \alpha \wedge \varphi+2 \pi \int_{S} K d S+2 \int_{C \ltimes C} \theta \sin \theta \frac{d x d y}{\|y-x\|^{2}} .
\end{aligned}
$$

It remains only to check that the contribution of $R_{\epsilon}$ vanishes as $\epsilon \rightarrow 0$. This is done in the next subsection.

4.5. Asymptotic estimations. Next we construct a singular chain $R_{\epsilon}$ in $\partial \Delta_{\epsilon}^{\prime}$ with $\partial R_{\epsilon}=-\partial T_{\epsilon}$ as promised. Let $v: \partial \Delta_{\epsilon} \rightarrow E$ be the section given by the vertical planes. With the same kind of arguments as in the proof of Proposition 14 one shows that $\tau \circ v$ lifts over $q$; i.e. there exists $h: \partial \Delta_{\epsilon} \rightarrow \mathbb{R}$ such that $q(x, h(x))=\tau \circ v(x)$. Let $R_{0, \epsilon} \subset \partial \Delta_{\epsilon}^{\prime}$ be the graph of $h$ over $\partial \Delta_{\epsilon}$. In particular, both $T_{1} \cap \partial \Delta_{\epsilon}^{\prime}$ and $\partial R_{0, \epsilon}$ project by $\pi$ onto $\partial B \cap \partial \Delta_{\epsilon}$. Next we consider the union of vertical segments joining these two graphs. More precisely, we define 
$R_{1, \epsilon}=\left(C \ltimes S \cap \partial \Delta_{\epsilon}\right) \times[0,1], R_{2, \epsilon}=\left(S \ltimes C \cap \partial \Delta_{\epsilon}\right) \times[0,1], R_{3, \epsilon}=\left(T^{+} S \cap \partial \Delta_{\epsilon}\right) \times[0,1]$ together with the mappings

$$
\begin{aligned}
\sigma_{i}: R_{i, \epsilon} & \rightarrow \partial \Delta_{\epsilon}^{\prime} \\
(z, t) & \mapsto\left(z, t g_{i}(z)+(1-t) h(z)\right),
\end{aligned}
$$

for $i=1,2$. As for $\sigma_{3}$, we take the same definition with $g$ in the place of $g_{i}$. We think of $\left\{R_{i, \epsilon}, i=0,1,2,3\right\}$ as singular chains in $\partial \Delta_{\epsilon}^{\prime}$, and we define $R_{\epsilon}=$ $\sum_{i=0}^{3} R_{i, \epsilon}$. A careful study of the boundaries shows that $\partial R_{\epsilon}=-\partial T_{\epsilon}$.

To finish the proof of Theorem 1 we only need to establish the following.

\section{Proposition 17.}

$$
\lim _{\epsilon \rightarrow 0} \int_{R_{\epsilon}} \pi^{*} \alpha \wedge \varphi=0
$$

Proof. Here we assume that $S$ coincides with the cylinder $C \times(0, \infty) \subset \mathbb{H}^{3}$ in a neighborhood of infinity. This is no loss of generality by Corollary [13, and equation (25).

In particular we may assume that $h$ and $g$ coincide over $T^{+} S$. Hence, the integral over $R_{3, \epsilon}$ vanishes. Next we concentrate on $R_{1, \epsilon}$ (the study of $R_{2, \epsilon}$ being obviously symmetric). Given $(x, y) \in C \times S \cap \partial \Delta_{\epsilon}$, let $\{x, z\}$ be the ideal endpoints of $\Phi(x, y)$. The euclidean distance between $x$ and $z$ is constant $\epsilon$. Hence, given $x \in C$ the point $z$ is determined by the angle $\gamma$ between the straight segment $\overline{x z}$ and $T_{x} C$. This angle $\gamma$ coincides with the length of the fiber interval $\pi^{-1}(x, y) \cap R_{1, \epsilon}$. By Fubini's theorem

$$
\int_{R_{1, \epsilon}} \pi^{*} \alpha \wedge \varphi=\int_{\pi\left(R_{1, \epsilon}\right)} \gamma \cdot \alpha=\int_{\pi\left(R_{1, \epsilon}\right)} \gamma \cos \gamma \frac{d x d \gamma}{\epsilon}
$$

since $\alpha=\cos \gamma \epsilon^{-1} d x d \gamma$ (cf. (14) ). The previous integrals vanish when $\epsilon \rightarrow 0$ since $\gamma=O(\epsilon)$. Indeed, the chords of length smaller than $\epsilon$ make angles with $C$ of order $O(\epsilon)$.

It remains to estimate the integral over $R_{0, \epsilon}$. Let $\left(x^{\prime}, y^{\prime}\right) \in S \ltimes S$ be a generic point in $M_{S} \cap \partial \Delta_{\epsilon}$. Let $x, y \in C$ be the vertical projections of $x^{\prime}, y^{\prime}$. Let $z, w$ be the ideal endpoints of the geodesic $\ell=\Phi\left(x^{\prime}, y^{\prime}\right)$. We choose Euclidean coordinates on $\mathbb{R}^{2} \equiv \partial_{\infty} \mathbb{H}^{3}$ so that $x_{2}=y_{2}=0$. We can assume $z_{1}<x_{1}<y_{1}<w_{1}$. Then

$$
\begin{gathered}
d z_{2}=(t+\sigma) \frac{d x_{2}}{\sigma}-t \frac{d y_{2}}{\sigma} \\
d w_{2}=(\sigma+t-\epsilon) \frac{d x_{2}}{\sigma}+(\epsilon-t) \frac{d y_{2}}{\sigma} .
\end{gathered}
$$

where $t=x_{1}-z_{1}, \sigma=y_{1}-x_{1}$, and thus $w_{1}-y_{1}=\epsilon-t-\sigma$. Recall that $\left(x^{\prime}, y^{\prime}\right)$ corresponds (through $\Phi^{\prime} \circ \tau^{-1} \circ \pi$ ) to the pair $(\ell, \wp)$ where $\wp$ is the vertical plane containing $\ell$. We take an adapted orthonormal frame $\left(p ; g_{1}, g_{2}, g_{3}\right)$ such that $p \in \ell$ 
projects vertically onto $\frac{1}{2}(z+w) \in \partial_{\infty} \mathbb{H}^{3}$ and $g_{3} \perp \wp$. Then (17) and the equations above yield

$$
\varphi=\left\langle\nabla g_{2}, g_{3}\right\rangle=\theta_{23}=\frac{1}{\epsilon}\left(d z_{2}+d w_{2}\right)=\frac{1}{\epsilon \sigma}\left((2 t+2 \sigma-\epsilon) d x_{2}+(\epsilon-2 t) d y_{2}\right) .
$$

Since $\pi^{*} \alpha=d \varphi$ we get

$$
\pi^{*} \alpha=\frac{2}{\epsilon \sigma}\left(d t \wedge d x_{2}-d t \wedge d y_{2}\right)-\frac{2 t-\epsilon}{\epsilon \sigma^{2}} d \sigma \wedge d x_{2}-\frac{\epsilon-2 t}{\epsilon \sigma^{2}} d \sigma \wedge d y_{2} .
$$

Hence, recalling that $x, y$ are restricted to move along $C$, we get

$$
\pi^{*} \alpha \wedge \varphi=\frac{4}{\epsilon^{2} \sigma} d t \wedge d x_{2} \wedge d y_{2}=\frac{4}{\epsilon^{2}} \sin \beta_{x} \sin \beta_{y} \frac{d t \wedge d x \wedge d y}{\|y-x\|},
$$

where $d x, d y$ denote arc-length elements on $C$, and $\beta_{x}, \beta_{y}$ are angles between $C$ and the segment $\overline{x y}$. Therefore

$$
\int_{R_{0, \epsilon}} \pi^{*} \alpha \wedge \varphi=\frac{4}{\epsilon^{2}} \int_{x, y \in C,\|y-x\| \leq \epsilon} \sin \beta_{x} \sin \beta_{y}(\epsilon-\|y-x\|) \frac{d x \wedge d y}{\|y-x\|}
$$

which goes to zero when $\epsilon \rightarrow 0$, since $\beta_{x}, \beta_{y}=O(\|y-x\|)$.

\section{REFERENCES}

[1] T.Banchoff, W.Pohl, A generalization of the isoperimetric inequality. J.Differential Geom. 6 (1971), 175-192.

[2] F.Dillen, W.Kühnel, Total curvature of complete submanifolds of euclidean space. Tohoku Math.J., 57 (2005), 171-200.

[3] N.Dutertre, A Gauss-Bonnet formula for closed semi-algebraic sets. Advances in Geometry 8 (2008), 33-51.

[4] J.Franklin, Some Stereological Principles in Morphometric Cytology. SIAM J.of App. Math., 33, n.2 (1977), 267-278.

[5] R.Langevin, J.O'Hara, Conformally invariant energies of knots. Journal of the Inst. of Math. Jussieu, 4 (2) (2005), 219-280.

[6] R.Langevin, G.Solanes, On bounds for total absolute curvature of surfaces in hyperbolic 3space. C. R. Math. Acad. Sci. Paris 336 (2003), no. 1, 47-50.

[7] J.M.Lee, Introduction to smooth manifolds. Graduate Texts in Math. 218, Springer-Verlag, New York, 2003.

[8] W.Pohl, Some integral formulas for space curves and their generalization. Amer.J. Math., 90, n.4 (1968), 1321-1345.

[9] L.A.Santaló, On some invariants under similitudes for convex bodies. Discrete geometry and convexity, Ann. New York Acad. Sci., 440, 128-131, New York Acad. Sci., 1985,

[10] L.A.Santaló, Integral geometry and Geometric Probability. Cambridge University Press, Cambridge, 2004.

[11] R.Schneider, Convex bodies: the Brunn-Minkowski theory. Cambridge University Press, Cambridge, 1993.

[12] G.Solanes, Integral geometry and the Gauss-Bonnet theorem in constant curvature spaces. Trans. Amer. Math. Soc. 358 (2006), no. 3, 1105-1115. 
[13] E.Teufel, Isoperimetric inequalities for closed curves in spaces of constant curvature. Results in Math., 22 (1992), 622-630.

[14] H.Whitney, The self-intersections of a smooth n-manifold in $2 n$-space. Ann.of Math. 45 n.2 (1944), 220-246.

Departament de Matemàtiques, Univeristat Autònoma de Barcelona, Edifici CC. Campus de la UAB, 08193 Bellaterra

E-mail address: solanes@mat.uab.cat 